\title{
Microbiota diversity during neonatal colonization impacts gut physiology in a pig model
}

\author{
J. P. Lallès ${ }^{1}$, A. Bizon ${ }^{1}$, A. Taekema ${ }^{1}$, M. E. Arnal ${ }^{1}$, C. R. Stokes ${ }^{2}$, M. Bailey ${ }^{2}$, H. Smidt ${ }^{3}$, \\ A. Jansman ${ }^{4}$ and S. J. Koopmans ${ }^{3,4}$ \\ ${ }^{1} I N R A-A D N C$, Saint-Gilles, France, ${ }^{2}$ School of Veterinary Science, University of Bristol, Langford, Bristol BS40 5DU, \\ ${ }^{3}$ Wageningen University, Wageningen and ${ }^{4}$ Wageningen UR Livestock Research, Lelystad, The Netherlands
}

Reduced gut microbiota diversity is suspected to be detrimental in a variety of diseases, including inflammatory bowel diseases ${ }^{(1)}$. Variation in microbiota composition during gut colonization is known to influence neonatal mortality and may be a risk factor for various non-infectious diseases such as allergy, metabolic disorders and obesity later in life ${ }^{(2)}$. How neonatal microbiota diversity can modulate gut barrier function and defense systems is poorly understood. Therefore, the aim of the present study was to test the hypothesis that a complex microbiota is more suitable neonatally than a simplified microbiota for homeostatic development of gut function.

This hypothesis was tested in a neonatal pig model (Lelystad ethics protocol No. 2011097). Piglets were delivered by Caesarean section and were kept in SPF facilities. They were then administered orally at day 2 and 3 a simple microbiota (SM, $n=6)$ alone or with a faecal bacterial suspension from an unrelated sow at day $4(\mathrm{CM}, n=6)$. The three bacterial genera and strains of the SM mixture ('Bristol mix') were recently shown to reliably colonize the gut of germfree pigs ${ }^{(3)}$. The piglets were slaughtered at 16 days of age. Segments of proximal jejunum, distal ileum and proximal colon and plasma were collected. Gut samples were analysed for villus-crypt morphology, enzyme activities (intestinal alkaline phosphatase, IAP; dipeptidyl-peptidase IV; sucrase), inducible heat shock proteins, soluble protein and IAP in digesta. Four plasma markers of inflammation (C-reactive protein, haptoglobin, TNF- $\alpha$ and $\alpha$-acid glycoprotein) were analysed. Treatment effects were analysed by T-test.

At day 16, body weights and diversity of intestinal microbiota did not differ between the groups. However, an aberrant composition was observed in the jejunum and ileum of SM pigs [lower abundance of several presumed beneficial microbial groups (lactobacilli, butyrogenic species) and increase in a number of potential pathogens]. Jejunal crypts were deeper $(P<0.05)$, ileal surface area tended to be larger $(P=0.080)$ and colon crypts tended to be narrower $(P=0.056)$ in $\mathrm{CM}$ pigs. Sucrase activity in the jejunal mucosa was lower in $\mathrm{CM}$ pigs $(P<0.05)$ (other enzymes in jejunal and ileal tissue unaffected). Soluble protein and IAP activity were lower in ileal $(P<0.05$, both) and colonic $(P<0.05$ and $P<0.01$, respectively) digesta of CM pigs. Finally HSP70 relative concentration tended $(P=0.096)$ to be lower in jejunal tissue. HSP27 tended to be lower in the ileum $(P=0.064)$ but was higher in the colon $(P<0.05)$ of $\mathrm{CM}$ pigs. Systemic inflammation did not differ between treatments.

Neonatal microbiota complexity profoundly affected various aspects of gut tissue and digesta characteristics, according to distinct regional patterns in pigs. $\mathrm{CM}$ reduced jejunal maturity, tended to reduce bacterial-induced stress on the intestine but increased it on the colon ${ }^{(4)}$. Finally, gut luminal degradation potential was higher in CM pigs.

1. Berry D \& Reinisch W (2013) Best Pract Res Clin Gastroenterol 27, 47-58.

2. Matamoros S, Gras-Leguen C, Le Vacon F et al. (2013) Trends Microbiol 21, 167-173.

3. Laycock G, Sait L, Inman C et al. (2012) Vet Immunol Immunopathol 149, $216-224$.

4. Arvans DL, Vavricka SR, Ren H et al. (2005) Am J Physiol Gastrointest Liver Physiol 288, G696-G704. 